\title{
Iniciação e participação no basquetebol: ensino da fase ofensiva a partir de conceitos de jogo
}

\author{
Sampling years and participation in basketball: teaching the offensive phase from game concepts
}

Iniciación y participación en baloncesto: enseñanza de la fase ofensiva basada en conceptos de juego

\author{
Yura Yuka Sato dos Santos \\ Faculdade de Educação Física. Universidade Estadual de \\ Campinas, Brasil \\ yura_sato@hotmail.com \\ (D) https://orcid.org/0000-0002-9422-6953
}

\section{Lucas Marques Maricone}

Faculdade de Ciências Aplicadas. Universidade Estadual de

Campinas, Brasil

lucasmaricone@hotmail.com

(iD https://orcid.org/0000-0002-2164-9963

\section{Bartira Pereira Palma}

Faculdade de Educação Física. Universidade Estadual de

Campinas, Brasil

bartirapalma@gmail.com

(D) https://orcid.org/0000-0002-7648-8060

\author{
Larissa Rafaela Galatti \\ Faculdade de Ciências Aplicadas e Faculdade de Educação \\ Fisica. Universidade Estadual de Campinas, Brasil \\ lagalatti@hotmail.com \\ (iD https://orcid.org/0000-0003-1743-6356
}

\section{Resumo:}

Os jogos de ataque por conceitos (JAC) visam estimular jogadores a compreenderem o jogo, solucionarem problemas e criarem vantagens sobre o adversário. O objetivo foi aplicar os JAC para o ensino da fase ofensiva do basquetebol no esporte de participação brasileiro, descrevendo as opiniões dos atletas e seu treinador. Participaram oito atletas do sexo masculino (média de 16,8 $\pm 1,0$ anos), com tempo médio de experiência com o basquetebol de 3,0 \pm 1,4 anos, com métodos tradicionais de ensino, e um treinador de 32 anos com oito anos de experiência profissional. Elaboramos os JAC a partir dos conceitos: dividir e passar, passar e cortar, e jogo ao poste. Aplicamos oito sessões de treino, com duração de 90 minutos cada. Realizamos entrevista semiestruturada com o treinador e grupos focais com os atletas, analisadas por análise temática. Nos resultados estabelecemos as categorias opiniões positivas (temas: compreensão da lógica do jogo; aspectos pessoais; e aspectos interpessoais), e negativas (temas: confronto com o novo e tempo de implementação do método). Os JAC foram compreendidos como prazerosos e facilitaram a compreensão da lógica do jogo. Concluimos que os JAC podem ser utilizados para ensino do basquetebol no contexto de participação, com vantagens sobre abordagens pedagógicas tradicionais.

PalaVras-Chave: Abordagens Pedagógicas, Prática Esportiva, Iniciação Esportiva Tardia. 


\begin{abstract}
:
Attack games through concepts (JAC) aim at stimulating players to understand the game, solve problems and create advantages over the opponent. The objective of this study is to apply JAC to teach the basketball offensive phase in the context of sports participation, describing the perceptions of Brazilian athletes and their coach. The participants were eight male athletes (aged $16.8 \pm 1.0$ ), with an average experience in basketball of $3.0 \pm 1.4$ years, based on traditional teaching methods, and a 32-yearold coach with eight years of professional experience as such. We elaborated JACs based on the following concepts: cut and pass, give and go, and post-game. We applied eight training sessions of 90 minutes each. We conducted a semi-structured interview with the coach and focus groups with the athletes. Data was analyzed through thematic analysis. In the results, we established the categories for positive opinions (themes: understanding the game logics, personal aspects, and interpersonal aspects), and for the negative ones (confronting the unknown and method implementation time). JAC seemed to be a pleasant practice and facilitated the understanding of the game's logics. We conclude that JAC can be used to teach basketball in the context of participation, with advantages over traditional pedagogical approaches.
\end{abstract}

KEYWORDS: Pedagogical approaches, Sports practice, Late sports initiation.

\title{
Resumen:
}

Los juegos de ataque por conceptos (JAC) tienen como objetivo alentar a los jugadores a comprender el juego, resolver problemas y crear ventajas sobre el oponente. El objetivo fue aplicar el JAC para la enseñanza de la fase ofensiva del baloncesto en el deporte participativo brasileño, describiendo las opiniones de los atletas y su entrenador. Participaron ocho deportistas masculinos (media 16,8 \pm 1,0 años), con una experiencia media con el baloncesto de 3,0 \pm 1,4 años, con métodos de enseñanza tradicionales, y un entrenador de 32 años con ocho años de experiencia profesional. Construimos los JAC alrededor de los conceptos: dividir y pasar, pasar y cortar, y juego al poste. Aplicamos ocho sesiones de entrenamiento de 90 minutos cada una. Realizamos entrevistas semiestructuradas con el entrenador y grupos focales con los deportistas, analizados mediante análisis temático. En los resultados se establecieron las categorías opiniones positivas (temas: comprensión de la lógica del juego; aspectos personales; y aspectos interpersonales), y negativos (temas: confrontación con lo nuevo y tiempo de implementación del método). Los JAC se entendieron como placenteros y facilitaron la comprensión de la lógica del juego. Concluimos que se puede utilizar los JAC para enseñar baloncesto en el contexto de la participación, con ventajas sobre los enfoques pedagógicos tradicionales.

Palabras Clave: Enfoques Pedagógicos, Práctica Deportiva, Iniciación Deportiva Tardía.

\section{INTRODUÇÃO}

O basquetebol está no grupo dos Jogos Esportivos Coletivos (JEC), considerados jogos complexos devido à interação de diferentes fatores, que devem ser administrados de forma precisa e em tempo adequado para que as ações culminem em um resultado eficaz (Moreira \& Paes, 2011; Robles, Benito, Fuentes-Guerra, \& Rodríguez, 2013). Isso exige de quem joga cooperar com seus colegas de time, estabelecer uma estratégia comum de jogo, lidar com sua imprevisibilidade, leitura tática das situações que emergem, além da execução de ações técnicas adequadas para a resolução dos problemas do jogo. Em especial, o basquetebol contempla restrições de tempo que obrigam o jogador a realizar essas ações sob pressão, o que torna a tomada de decisão uma variável essencial para o sucesso no jogo (Ibañez, Garcia-Rubio, Gómez, \& Gonzalez-Espinosa, 2018; Silva, Galatti, \& Paes, 2010). Devido a essas características, altos níveis de proficiência na modalidade somente são atingidos após anos de prática. $\mathrm{Na}$ tentativa de acelerar esse processo muitos treinadores especializam atletas em uma modalidade específica, ou em posições e funções da modalidade, em fases iniciais do desenvolvimento esportivo (Galatti et al., 2016; Menezes, Marques, \& Nunomura, 2014), reduzindo, assim, a quantidade de variáveis com que o atleta precisa lidar. Nesse cenário, a simplificação do jogo, como limitar um atleta a uma função específica, ou a tentativa de ensinar respostas prontas, pode impedir o comportamento criativo e comprometer a tomada de decisão.

O ensino dos JEC por meio de exercícios descontextualizados das situações de jogo ainda é comum. Ford, Yates, e Williams (2010) observaram que treinadores de crianças e adolescentes tendem a usar a prática deliberada no processo de treinamento de suas equipes. A prática deliberada se pauta em abordagens pedagógicas tradicionais e consiste no treinamento sistematizado focado no aprimoramento técnico, demasiadamente rígido e geralmente focado em fatores motivacionais extrínsecos, como o resultado em competições, o que pode prejudicar o aprendizado e a motivação intrínseca de atletas (Côté, Baker, \& Abernethy, 2003, 2007). As abordagens tradicionais (tecnicistas) se baseiam em tarefas não representativas, 
que dissociam a informação do ambiente da ação. Nesse sentido, desconsidera-se os elementos estruturais e funcionais do contexto de jogo, imprescindíveis para o desenvolvimento da competência nos JEC (Scaglia, Reverdito, Leonardo, \& Lizana, 2013). Portanto, o foco das abordagens tradicionais no ensino das habilidades técnicas, com estratégias analíticas e descontextualizadas, tomando como modelo padrões de movimento de atletas de elite, o que desfavorece a compreensão do jogo e não estimula as tomadas de decisões, além de poder ser desmotivante (Bunker \& Thorpe, 1982; Galatti, Reverdito, Scaglia, Paes, \& Seoane, 2014; Galatti, Seoane, \& Paes, 2012; Gray \& Sproule, 2011; Robles et al., 2013; Travassos et al., 2012).

Com o objetivo de preencher as lacunas das abordagens tradicionais de ensino dos JEC, alguns modelos de iniciação e formação esportivas têm sido propostos na literatura em Pedagogia do Esporte (PE) nas últimas décadas (Bunker \& Thorpe, 1982; Greco, 2006; Kirk \& MacPhail, 2002; Light \& Tan, 2006; Paes, 2001; Reverdito, Galatti, Scaglia, \& Paes, 2020; Turner \& Martinek, 1995). Esses modelos se sustentam na teoria interacionista do conhecimento e se apoiam no princípio global-funcional, tendo o jogo como principal meio (Galatti et al., 2017; Leonardo, Scaglia, \& Reverdito, 2009; Rigon, Novaes, \& Tsukamoto, 2020). Nesse contexto, o planejamento de práticas com ênfase na manipulação das possibilidades de ação dos atletas visam o estímulo à criatividade e ao comportamento exploratório, melhorando a capacidade de percepção das fontes críticas de informação no ambiente que, por sua vez, estimulam a tomada de decisão (Vaughan, Mallett, Davids, Potrac, \& López-Felip, 2019). Por isso, alguns estudos têm demonstrado que a diversidade de práticas esportivas e de ocupação de funções na modalidade antes da especialização pode facilitar o aprendizado da modalidade, além de maximizar o impacto que a atividade esportiva tem no desenvolvimento dos jovens, contribuindo para a manutenção da prática em longo prazo (DiFiori et al., 2018; Fraser-thomas, Côté, \& Deakin, 2005; Galatti et al., 2016).

A Associação Nacional de Basquetebol (NBA) da América do Norte e a Organização Americana de Basquetebol (USA Basketball) reuniram especialistas para compartilhar suas pesquisas aplicadas e práticas acerca da participação de crianças e jovens no esporte, além de desenvolver um guia de recomendações para promover uma experiência positiva e saudável no basquetebol (DiFiori et al., 2018). Dentre as sete recomendações acerca da participação, destacamos três: a) os jovens devem ser incentivados a se envolver em diversos esportes, e adiar a especialização em um único esporte, especialmente antes dos 14 anos; b) a promoção do envolvimento pessoal deve ser uma prioridade, de forma que os jovens se conectem com outras pessoas, construam relacionamentos e assumam desafios e papéis de liderança, pois isso contribui para o desenvolvimento pessoal geral, bem-estar e desenvolvimento de talentos; e c) os jovens devem ser encorajados a se envolverem em atividades organizadas, lideradas por um treinador e também por colegas. De acordo com os especialistas do estudo, o jogo liderado por colegas permite que os jovens vivenciem atividades autodeterminadas e intrinsecamente motivadas, sejam criativos e se desafiem. Isso pode contribuir para o crescimento individual e desenvolvimento de talentos em longo prazo.

Alguns autores defendem que na iniciação esportiva e no contexto de participação/recreação os atletas deveriam ter mais oportunidades de praticar o jogo deliberado (prática gerida pelos próprios atletas, intrinsecamente motivada) do que a prática deliberada (Côté et al., 2003, 2007). Entretanto, Berry, Abernethy, e Côté (2008) observaram que não foi o envolvimento com a prática deliberada ou o jogo deliberado durante a formação que diferenciou atletas profissionais mais habilidosos em tomar decisões no jogo, mas sim o grau de envolvimento em atividades com demandas perceptuais e de tomada de decisão, ou seja, atividades baseadas em jogos. Isso destaca a necessidade de as rotinas de treinamento (prática estruturada) estarem focadas em atividades centradas no jogo. Desta forma, considerando que um(a) atleta em formação passará grande parte de seu tempo de prática esportiva em atividades geridas por um(a) treinador(a), essas atividades devem ser pautadas nas abordagens mais atuais da PE, organizadas para a promoção de uma experiência esportiva positiva e diversificada para as crianças e jovens.

Com o objetivo de balizar uma proposta de iniciação e formação no basquetebol pautados no princípio global-funcional, Maricone, Santos, Pérez, e Galatti (2016) propõem o “jogo de ataque por conceitos”. 
Conceitos no basquetebol são normas de aplicação às situações de jogo (Zoilo, 2008), de forma que os jogadores possam decidir sobre o que e como fazer em função das ações da defesa e dos companheiros (Rivero, n.d.). Essa proposta foi construída com base no método da Federação Espanhola de Basquetebol (FEB), documentada no website do clube do treinador da FEB. A principal premissa desse método é ensinar a jogar em qualquer área da quadra e exercer diversas funções, estimulando a criação de vantagens sobre o adversário. A divisão da movimentação ofensiva se pauta, basicamente, em apenas duas diferenciações de função, o jogador com bola e jogadores sem a bola, que podem ser exercidas por qualquer jogador em quadra. Dessa forma, não há especialização de movimentos e funções no jogo, essencial para o treinamento do jovem no basquetebol (DiFiori et al., 2017, 2018). Essa proposta visa dar suporte aos treinadores em sua atuação prática, com potencial de contribuir para o preenchimento da lacuna relativa à distância entre teoria e prática no que concerne aos modelos teóricos de iniciação e formação esportivas. Entretanto, esse é um modelo baseado em uma proposta de outro país, que ainda não foi investigado no contexto brasileiro. Dessa forma, é necessário conhecer sua aplicabilidade e compreender quais as opinióes de treinadores e atletas sobre o processo de ensino-aprendizagem baseado no modelo.

Destaca-se que as abordagens pedagógicas e modelos teóricos discutidos são independentes de faixa etária, dado que a iniciação esportiva é um fenômeno que pode ocorrer em qualquer idade. Poucos estudos abordam a iniciação esportiva em outras fases da vida que não a infância, mais especificamente no esporte de participação. Essa é uma lacuna importante na literatura visto que a prática esportiva regular pode promover o desenvolvimento de competências esportivas, socioemocionais e beneficios à saúde (DiFiori et al., 2018; Fraser-thomas et al., 2005). Os participantes deste estudo são adolescentes que inicaram a prática tardiamente no âmbito do esporte de participação. Investimentos de pesquisa nesta área são necessarios devido ao expresivo número de participantes nesse contexto, caracterizando-se como uma das maiores áreas de atuação do profissional de educação física no esporte e, por isso, com grande potencial de impacto social positivo. Com base no exposto, esta pesquisa tem como principal objetivo a aplicação de jogos de ataque por conceitos (Maricone et al., 2016) para o ensino da fase ofensiva do basquetebol no contexto do esporte de participação, assim como descrever as opinióes dos jovens atletas e treinador participantes desse processo em contexto brasileiro.

\section{Metodologia}

\section{Participantes}

Participaram do estudo oito atletas (16,8 $\pm 1,0$ anos, sexo masculino) com tempo médio de prática de três anos ( $\pm 1,4$ anos), e um treinador de 32 anos com oito anos de experiência em treinamento em basquetebol e dez anos em arbitragem. Os atletas e o treinador fazem parte de um programa gratuito de treinamento de basquetebol para crianças e jovens, da prefeitura da cidade de Limeira/São Paulo, no âmbito da participação esportiva, incluindo a iniciação esportiva tardia. Os atletas treinam duas vezes por semana, em sessões de 1h30min. A escolha dos participantes se deu pelos critérios de amostragem por conveniência (Patton, 2002), de acordo com a proximidade, disponibilidade e determinação em participar do estudo. Embora os jovens participantes tivessem em média três anos de contato com a modalidade, as experiências prévias dos atletas eram baseadas em métodos tecnicistas (descrição no subcapítulo a seguir), o que permitiu com que eles percebessem diferenças e/ou similaridades entre as situações de treinamento já vivenciadas na modalidade com a proposta de treinamento deste estudo.

A pesquisa teve aprovação pelo Comitê de Ética em Pesquisa institucional, com o número do CAAE: 82787617.2.0000.5404. A implementação dos treinos foi autorizada pela Secretaria de Esporte e Lazer da cidade de Limeira/São Paulo, responsável legal pelo programa de treinamento. Os participantes e seus 
responsáveis legais assinaram o Termo de Consentimento Livre e Esclarecido. Os atletas com menos de 18 anos assinaram o Termo de Assentimento para Crianças e Adolescentes. Todos os procedimentos da pesquisa foram explicados pessoalmente pelo pesquisador responsável aos participantes. Os treinos foram conduzidos pelo segundo autor desta pesquisa, contudo, o treinador da equipe acompanhou todos os treinos.

\section{Experiências prévias dos atletas participantes}

Aplicamos um questionário, elaborado pelos autores, a fim de investigar as experiências de treinos vivenciadas anteriormente pelos atletas, assim como compreender a concepção sobre um "treino ideal" na visão dos deles. Esses dados foram importantes para a interpretação das percepções dos participantes acerca da prosposta deste estudo. Os atletas constataram que as experiências prévias eram baseadas em treinos analítico-sintéticos, com divisão das partes para o todo, ou seja, divisão de partes do jogo em exercícios de fundamentos técnicos e físico, por exemplo. O princípio analítico-sintético tem como pressupostos: (a) partir do conhecido ao desconhecido - das partes ao todo; (b) do fácil para o difícil - diminuição da ajuda; c) do simples para o complexo - aproximação gradativa; e d) divisão do movimento em fases funcionais (Galatti, Paes, \& Seoane, 2009; Greco, 1998). Nesse sentido, o atleta A1 descreveu que, em sua experiência, os treinos consistiam em "aquecimento, físico, fundamentos e coletivos no final". O atleta A4 também relatou: "O aquecimento consistia em corrida batendo bola, de costas, corrida lateral, etc. $\mathrm{O}$ (trabalho) físico era com abdominais, agachamentos e treino de equilíbrio. E o (trabalho de) fundamentos era passe, etc."

A concepção de um treino ideial na visão dos atletas, por sua vez, também se baseia no princípio analíticosintético, como relata o atleta A3: "o treino ideal é revezado em físico, jogadas e coletivo". Dado que as experiências se resumem principalmente em treinos analíticos, dificilmente os atletas projetariam outros métodos como ideais em suas concepções.

\section{Procedimentos metodológicos e instrumentos utilizados}

O estudo seguiu procedimentos da pesquisa qualitativa e se caracterizou por uma pesquisa-ação, seguindo as ações de (i) planejamento, (ii) implementação e (iii) avaliação (Tripp, 2005) de conteúdos de ensinotreino para iniciação ao basquetebol.

\section{Planejamento das sessões de treino}

Na etapa de (i) planejamento do treinamento, elaboramos jogos de ataque por conceitos (JAC) baseados nos seguintes conceitos do método da FEB, descritos por Maricone et al. (2016): dividir e passar (Figura 1), passar e cortar (Figura 2) e jogo ao poste (Figura 3). Optamos pelos três conceitos para que os atletas não fossem sobrecarregados com muitas informações novas em um curto período de implementação. Para cada JAC descrevemos a sua matriz, de acordo com Scaglia, Reverdito e Galatti (2013), os referenciais estruturais, apoiados em Bayer (1994), e os fundamentos técnicos principais envolvidos, embasados em Galatti et al. (2017). De acordo com Menezes, Marques, e Nunomura, (2014), os treinadores devem viabilizar o ensino dos jogos esportivos coletivos através de jogos e brincadeiras que motivem a continuidade dos jovens no esporte. Desta forma, desenvolvemos os JAC com jogos e brincadeiras tradicionais. Ressaltamos que os conceitos coexistem (Maricone et al., 2016), logo, o fato de ter um conceito como dado objetivo principal não elimina a presença de outros conceitos nos JAC. Ademais, as estruturas dos JAC não são rígidas. Em todos os JAC o sistema defensivo adotado foi o individual. Os referenciais estruturais (espaço de jogo, número de jogadores, alvos, bolas e regras) podem e devem ser modificados de acordo com o objetivo e o grupo de treinamento (Galatti, Ferreira, Silva, \& Paes, 2008; Machado, Galatti, \& Paes, 2012). Além disso, os 
referenciais devem ser modificados no sentido de manter o jogo em nível ideal de desafio para os praticantes, afinal, deixando de haver desafio, o jogo deixará de ser jogo (Huizinga, 1999), bem como o ambiente deixará de ser de aprendizagem (Scaglia et al., 2013).

\section{FIGURA 1}

Descrição e esquema dos JAC sobre o dividir e passar.

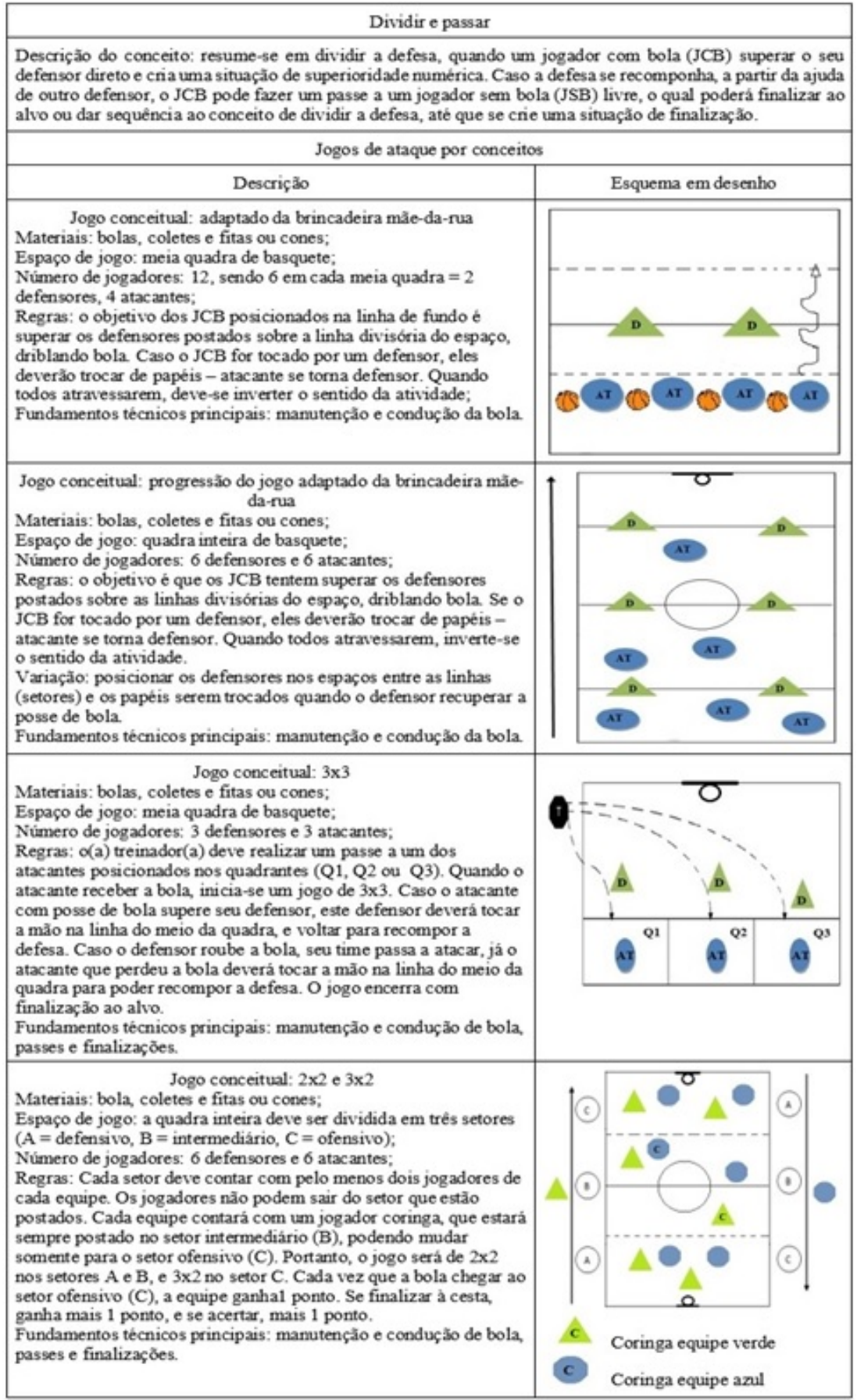


FIGURA 2

Descrição e esquema dos JAC sobre o passar e cortar

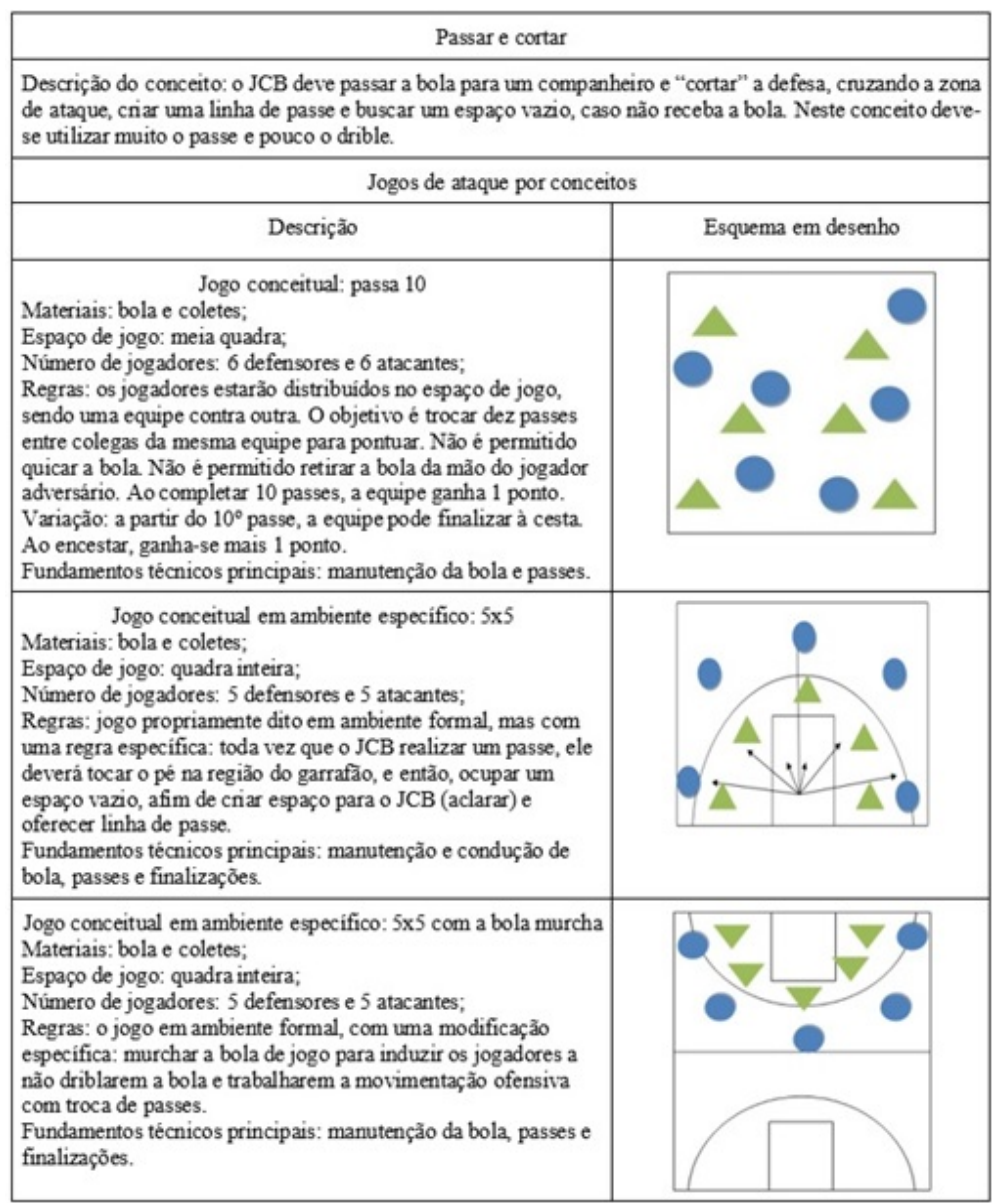

Fonte: elaboração própria

FIGURA 3

Descrição e esquema dos JAC sobre o jogo ao poste

\begin{tabular}{|c|c|}
\hline \multicolumn{2}{|c|}{ Jogo ao poste - triangulação } \\
\hline \multicolumn{2}{|c|}{$\begin{array}{l}\text { Descrição do conceito: o jogador que serve de referência ofensiva é o que se situa em poste médio, ou seja, } \\
\text { próximo à linha de lance livre, no meio da cabeça do garrafão. Busca-se este jogador de referéncia a fim de } \\
\text { criar triangulaçôes entre os jogadores internos e extemos ao perimetro, assim como criar espaços vazios, } \\
\text { sempre havendo mais de uma opção de passe para o JCB. }\end{array}$} \\
\hline \multicolumn{2}{|c|}{ Jogos de ataque por conceitos } \\
\hline Descrição & Esquema em desenho \\
\hline $\begin{array}{l}\text { Jogo conceitual: } 2 \times 2+1 \\
\text { Materiais: bola e coletes; } \\
\text { Espaço de jogo: meia inteira; } \\
\text { Número de jogadores: } 2 \text { defensores e } 2 \text { atacantes }+1 \text { apoio; } \\
\text { Regras: neste jogo haverá um poste fixo (que será uma } \\
\text { posição rotativa). Os jogadores de ataque deverão passar a } \\
\text { bola para o jogador posicionado no poste, para receberem } \\
\text { novamente em situação de finalização. Pode-se também variar } \\
\text { entre poste baixo, médio e alto. O jogador de poste não pode } \\
\text { finalizar. A finalização só poderá ocorrer após pelo menos um } \\
\text { passe ao poste. } \\
\text { Fundamentos técnicos principais: manutenção e condução da } \\
\text { bola, passes e finalizaçő́es. }\end{array}$ & nsores \\
\hline $\begin{array}{l}\quad \text { Jogo conceitual em ambiente especifico: } 5 \times 5 \\
\text { Materiais: bola e coletes; } \\
\text { Espaço de jogo: quadra inteira; } \\
\text { Número de jogadores: } 5 \text { defensores e } 5 \text { atacantes; } \\
\text { Regras: jogo em ambiente formal, no entanto, toda vez que um } \\
\text { atacante receber um passe dentro de uma das zonas de poste, } \\
\text { será somado } 1 \text { ponto ao placar geral. } \\
\text { Fundamentos técnicos principais: manutenção da bola, passes } \\
\text { e finalizações. }\end{array}$ & $\begin{array}{l}\text { Zonas de poste } \\
\text { Atacantes } \\
\text { Defensores }\end{array}$ \\
\hline
\end{tabular}

Fonte: elaboração própria 


\section{Implementação}

A (ii) implementação ocorreu entre maio e junho de 2018, em oito sessões de treino, com duração de aproximadamente uma hora e trinta minutos cada. Antes da implementação, o segundo autor deste estudo explicou ao treinador todos os conceitos de ataque do método FEB e o planejamento desenvolvido. No início de cada sessão o pesquisador também explicou aos atletas o objetivo e o conceito de ataque principal a ser trabalhado. Cada conceito foi implementado, por meio dos JAC, em duas sessões consecutivas e mais uma sessão de transição entre um conceito e outro (Figura 4). Desta forma, nas sessões três e seis foram trabalhados dois conceitos, como uma forma de introduzir o próximo conceito da fase de implementação.

FIGURA 4

Processo de implementação dos JAC nas oito sessões de treinamento

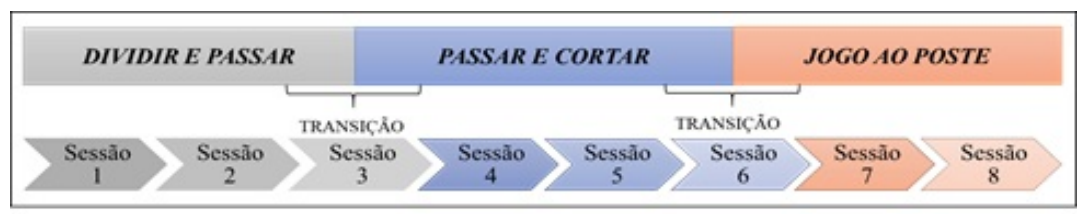

Fonte: elaboração própria

\section{Avaliação}

Para garantir a validade e confiabilidade dos dados (Patton, 2002), a (iii) avaliação se deu pela triangulação de diferentes métodos: investigamos as opinióes acerca do modelo implementado, na perspectiva do treinador, a partir de uma entrevista individual semiestruturada, e na perspectiva dos atletas, a partir de grupos focais (GF) (Hennink, 2014; Smith \& Sparkes, 2016). Os GF ocorreram em duas sessões com três e cinco atletas, respectivamente, devido à disponibilidade dos atletas. Uma segunda pessoa, também autora deste estudo, acompanhou o pesquisador nos GF, a fim de registrar os relatos e fazer perguntas aos entrevistados (Creswell, 2010). A entrevista semiestruturada foi gravada em áudio e os GF foram gravadas em áudio e vídeo, para posterior transcrição e uma análise mais fiel dos dados. As sessões de treino, assim como a aplicação do questionário, as entrevistas e os GF, foram realizados no local e horário de treinamento dos participantes.

\section{Análise de dados}

As transcrições da entrevista semiestruturada e dos grupos focais foram analisadas com base na análise temática seguindo as seis fases: familiarização, codificação, desenvolvimento temático, refinamento, nomeação e escrita (Braun \& Clarke, 2006; Braun, Clarke, \& Weate, 2016). O estabelecimento dos temas e sub-temas se deu pela análise de dois pesquisadores, de forma independente, e a confirmação se deu através de discussão e confrontamento entre os pesquisadores.

\section{Resultados e Discussão}

\section{Avaliação: opiniões dos participantes sobre o jogos de ataque por conceitos}

$\mathrm{Na}$ análise da entrevista com o treinador e os GF com os atletas estabelecemos duas categorias centrais, opiniões positivas e negativas. Os temas das opiniões positivas foram: a) a compreensão da lógica do jogo; b) aspectos pessoais; e c) aspectos interpessoais. Os temas das opiniões negativas foram: a) confronto com o novo; e b) tempo de implementação do método. Para cada tema também foram estabelecidos sub-temas (Figura 5). Falas representativas dos temas e sub-temas são apresentadas nesta sessão. 


\section{FIGURA 5}

Mapeamento dos temas e sub-temas sobre as opiniões dos participantes (atletas e treinador) acerca dos jogos de ataque por conceitos.

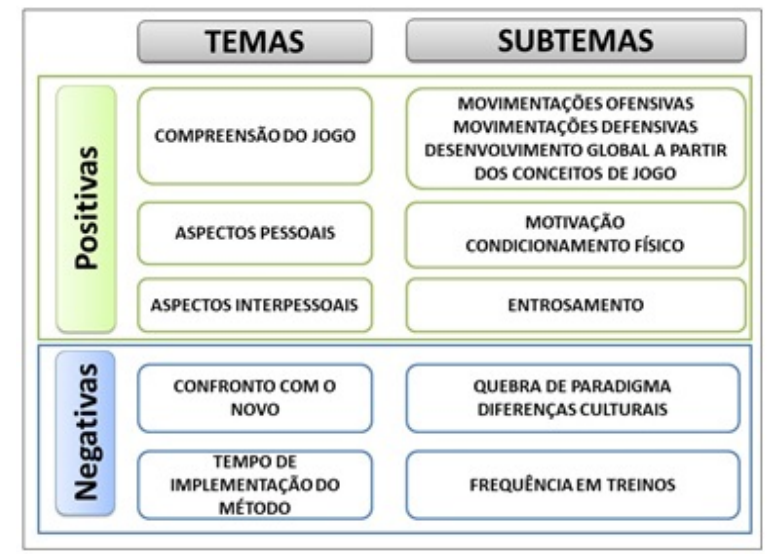

Fonte: elaboração própria

De acordo com a análise temática, nas opiniões positivas, tanto o treinador, quanto os atletas, relataram que o método facilita a compreensão da lógica do jogo, indicando que, mesmo com um período relativamente curto de implementação, os JAC se mostram interessantes para o ensino do basquetebol. Segundo Galatti et al. (2017) a lógica dos JEC se baseia na relação entre os referenciais estruturais e funcionais do jogo, com o objetivo principal de pontuar mais que o adversário. Dessa forma, os JEC podem ser considerados tarefas complexas (Vaughan et al., 2019), e entender sua lógica significa entender como seus elementos interagem, ou seja, ter uma percepção mais apurada das possibilidades de ação que o ambiente de jogo oferece, como falhas na defesa do adversário ou um colega de time livre para fazer a cesta, bem como apresentar condições para aproveitar essas possibilidades de ação (Araújo, Hristovski, Seifert, Carvalho, \& Davids, 2019). No ensino do esporte por meio de abordagens tradicionais é comum o ensino de ações técnicas desconectadas do jogo, para que o aluno, após acumular o aprendizado de todas as técnicas, consiga inseri-las no jogo. Dessa forma, ocorre uma simplificação da tarefa, uma vez que os elementos que a tornam complexa são retirados, como a imprevisibilidade característica da interação entre atacante e defensor. Essa simplificação dificulta a compreensão da lógica do jogo, uma vez que fontes de informação essenciais para a identificação dos problemas do jogo e adequada tomada de decisão estão ausentes (Araújo \& Bourbousson, 2016). O "jogo de ataque por conceitos" utilizado neste estudo, entretanto, prezou pela preservação dessa complexidade, por entender que ela é importante para o aprendizado da tomada de decisão e no aumento da criatividade dos atletas. A percepção de melhora na compreensão da lógica do jogo pelos participantes pode ser explicada por esses fatores.

A teoria da dinâmica ecológica (Araújo, Davids, \& Hristovski, 2006), postula que percebemos o ambiente como possibilidades de ação (i.e. affordances). As habilidades técnicas e táticas já desenvolvidas pelo jogador influenciarão na percepção dessas possibilidades. Nesse sentido, saber jogar significa ser proficiente em identificar e escolher boas oportunidades de ação, além de ter as habilidades necessárias para agir (Araújo et al., 2019). As possibilidades de ação no jogo de basquetebol somente ocorrerão no contexto de jogo. Ensinar um atleta a jogar implica em facilitar a percepção dessas possibilidades de ação, diretamente relacionadas à lógica do jogo. Assim, o modelo aplicado no presente estudo mantém a estrutura básica do jogo, facilitando a percepção dessas possibilidades através de restrições impostas no ambiente. Os atletas participantes dessa pesquisa já possuíam um histórico na modalidade, de forma que já apresentavam um aprendizado prévio. As sessões de treinamento aplicadas permitiram que os atletas utilizassem esse conhecimento prévio na percepção de possibilidades de ação no contexto de jogo. Nesse sentido, o atleta A4 relatou: 
Ah, eu melhorei bastante a movimentação, visão de movimentação, e assim... eu sempre ficava perdido [...] eu não sabia o que fazer...os “caras" cruzavam e eu pensava "o que eu faço aqui?”. Eu entendi que se um companheiro entrou (no perímetro) com a bola eu não posso ficar lá, porque meu marcador vai nele, eu tenho que sair. (Atleta A 4)

O intuito da proposta foi desenvolver as movimentações ofensivas por meio dos conceitos, assim, as movimentações defensivas não eram o objetivo imediato. Contudo, houve relatos de uma melhora na compreensão sobre aspectos defensivos, o que corrobora com a complexidade de atividades como os JEC, nas quais a interação entre os elementos componentes é parte da organização da tarefa (Galatti et al., 2017; Vaughan et al., 2019), o que torna a presença da interação um fator essencial para o aprendizado da lógica do jogo. Com isso, é possível que os atletas aprendam não só sobre aspectos ofensivos, mas sobre aspectos defensivos e, possivelmente, outros aspectos do jogo. Dessa forma, entendemos como essencial a presença de defensores no treinamento de movimentações ofensivas, como ocorreu no modelo aplicado neste estudo, o que garante a oportunidade de percepção de possibilidades de ação tanto para atacantes, quanto para defensores. O atleta A8 relatou que "o que se tornou mais fácil foi entender o que estava acontecendo no jogo". Nessa direção, o atleta A5 também comentou que "quem participou mais dos treinos conseguiu ter, "tipo assim", mais técnica de observar a movimentação ofensiva, tanto ofensiva quanto defensiva. Conseguiu ter mais percepção de posicionamento, [...] mais percepção de movimento em quadra".

O treinador ao se referir sobre as vantagens do método fez menção a não especialização de posições:

Os movimentos específicos (das posições no basquetebol) foram poucos trabalhados, que as vezes são necessários, mas que podem ser desenvolvidos numa idade superior. Se compararmos com equipes que disputam campeonatos, isso é um fator que complica um pouco, pois acaba colocando em desvantagem. Mas, essa desvantagem é momentânea porque futuramente haverá um desenvolvimento melhor desses atletas. Na nossa cultura há uma especialização um pouco mais precoce. [...] Tenho essa consciência também de que os atletas até uma determinada idade precisam desenvolver o basquetebol como um todo [...] acho que eles necessitam desse desenvolvimento global do jogo, para depois especificar as posições. (Treinador)

O segundo tema identificado, também na esfera positiva, está relacionado a aspectos pessoais, especificamente motivação e condicionamento físico. A Teoria da Autodeterminação define a motivação como direção e esforços empregados para se atingir um objetivo. A interação destes aspectos resulta no contínuo da autodeterminação, que reflete o grau em que os comportamentos são emitidos em função de motivadores internos ou externos, variando de amotivação a motivação intrínseca (Deci \& Ryan, 1985; Pelletier et al., 1995; Ryan \& Deci, 2000). Os atletas participantes desta pesquisa, apesar de estarem nos anos iniciais da prática do basquetebol, já apresentavam algum tempo de experiência (média de 3,0 $\pm 1,0$ anos), e a manutenção da prática indica que eles já estavam motivados, afastando-se da amotivação (ausência de razão para a prática esportiva). O discurso dos atletas aponta para uma aproximação com o que Pelletier et al. (1995) denominou de motivação intrínseca para experiências estimulantes (busca de excitação, prazer e divertimento) e motivação intrínseca para conhecer (ligada à curiosidade e busca de compreensão).

$\mathrm{O}$ ensino dos JEC baseado em jogos tem sido indicado na literatura por se caracterizar como uma experiência prazerosa, entre outros motivos (Galatti et al., 2017), e este estudo fortalece esta afirmação visto que os atletas perceberam as atividades como estimulantes e sentiram-se motivados para a prática. DiFiori et al. (2018) afirmam, baseados em relatórios de pesquisas internas realizadas pela National BasketBall Association (NBA) em seus programas de basquete para crianças e adolescentes que a principal razão relatada para a prática da modalidade era divertir-se, o que torna importante que os atletas percebam a prática como divertida a fim de se manterem nela por longo período de tempo. Dessa forma, os resultados deste estudo corroboram o uso dos JAC como estratégia que favorece a manutenção da prática do basquetebol em longo prazo. Além disso, os atletas parecem se aproximar também da motivação intrínseca para conhecer, uma vez que a utilização de uma estratégia nova pode ter instigado a curiosidade e a necessidade de busca pela compreensão. Parece-nos natural que isso ocorra a partir do contato com uma estratégia nova de ensino da modalidade, entretanto, a utilização de jogos como estratégia de ensino promove o contato constante com a imprevisibilidade e a dinamicidade, o que pode contribuir para a consolidação da motivação intrínseca. 
Não foi objetivo do treinamento empregado neste estudo melhorar o condicionamento físico dos atletas, então, não houve nenhuma avaliação específica de indicadores de capacidades físicas. Entretanto, esse foi um tema que emergiu no discurso dos participantes da pesquisa. $\mathrm{Na}$ abordagem tradicional as ações técnicas são praticadas em situações simplificadas, com ou sem oposição (Silva, 2007). Nessas situações, é comum a utilização de estratégias como filas, o que pode reduzir o tempo ativo de prática dos atletas. A utilização de jogos pode ter aumentado o tempo de prática dos atletas durante o treino, e incrementado a intensidade, e isso pode ter sido percebido como melhora do condicionamento físico pelos atletas. Além disso, após dois ou três anos praticando por meio da mesma abordagem, a inserção de um estímulo novo pode levar o organismo a uma adaptação (Bompa, 2002), o que pode ter influenciado a percepção dos atletas também. Entretanto, estudos que envolvam medidas diretas de condicionamento físico são necessários para confirmar tais colocações. Sobre isso, o atleta A5 disse sobre o condicionamento físico: “[...] o seu físico, que fica melhor para defender e atacar”, e o atleta A1, falou: “[...] eu consegui pegar mais condicionamento físico que antes eu não tinha tanto".

O último tema identificado na categoria dos positivos foi o relacionado aos aspectos interpessoais, com o sub-tema entrosamento, como destacou o atleta A7: "Eu vi o time sendo melhor, já tendo mais entrosamento um com o outro". O atleta A5 também relatou que, "chegou numa hora do treino, em uma sequencia de treinos, em que todo mundo estava fazendo muito bem tudo o que tava acontecendo, (A1 concorda com a cabeça), isso deixa até o treino mais interessante".

$\mathrm{Na}$ perspectiva da abordagem dinâmica ecológica, um grupo percebe possibilidades de ação no ambiente de forma compartilhada, ou seja, as possibilidades de ação de cada indivíduo não são independentes. Então, nos JEC o time treina a percepção de possibilidades de ação compartilhadas a partir de um objetivo comum, e isso contribui para que o time atue em sinergia (Araújo \& Bourbousson, 2016; Silva, Garganta, Araújo, Davids, \& Aguiar, 2013). Isso explica como, em um curto espaço de tempo, jogadores de um time conseguem responder em conjunto a um estímulos proveniente de um ambiente dinâmico (Silva et al., 2015). Nesse sentido, a melhora no entrosamento percebida pelos atletas e treinador vão ao encontro da abordagem dinâmica ecológica, uma vez que a estratégia pedagógica utilizada neste estudo tem como característica manter variáveis importantes que caracterizam os JEC. Esse é um princípio pedagógico importante que contribui para o treinamento da percepção de ações compartilhadas, uma vez que fontes de informação importantes permanecem na atividade, garantindo a semelhança com o contexto real de jogo (Araújo \& Bourbousson, 2016).

No que diz respeito aos aspectos negativos, um dos temas identificados foi o confronto com o novo, com o sub-tema quebra de paradigmas. Apesar de abordagens pedagógicas baseadas na compreensão da lógica do jogo serem discutidas desde a década de 1960 (Werner \& Almond, 1990), ainda é muito comum no Brasil a utilização de abordagens centradas no ensino de ações técnicas no ensino do basquetebol (Ferreira, Galatti, \& Paes, 2005; Morales \& Greco, 2007), o que pode ter contribuído para a opinião dos participantes de que o protocolo aplicado representa uma quebra de paradigma pessoal, já que representava uma experiência nova. Além disso, conforme mencionado, os atletas participantes tinham experiência com a prática do basquetebol em uma abordagem tradicional de ensino. Então, para esse grupo essa abordagem já era conhecida, podendo ser considerada como um estado de comportamento estável, e o contato com uma nova abordagem pode ter provocado uma instabilidade no sistema atleta-ambiente (Araújo et al., 2019), o que pode estar relacionado com o fato de os atletas terem percebido isso como algo negativo, visto que o ambiente tornou-se desconhecido e desconfortável. Dessa forma, oito sessões de treinamento parecem ter sido insuficientes para que os atletas atingissem outra fase de comportamento estável. O atleta A5 nos descreveu: "[...] foi algo bem confrontador, não é, a gente parar de pegar a bola e bater bola, pra colocar o time e fazer essa engrenagem rodar." O treinador também comentou: 
A primeira impressão é que fica um pouco confuso até para os atletas que não estão acostumados com essa troca de posições. Então, eles têm uma dificuldade maior de entendimento,visto que eles já estão acostumados e até com alguns vícios das posições determinadas que eles já atuaram.

O segundo tema expressa a opinião dos participantes de que a menor frequência de alguns alunos produziu um efeito negativo no trabalho desenvolvido pela equipe. $\mathrm{O}$ atleta $\mathrm{A} 3$ destacou:

[...] quem foi mais expressivo em treino, conseguiu entender a questão dos cinco na linha e a questão do passe no meio (poste médio) e infiltrar, só que quem foi menos expressivo no treino não deu esse respaldo para o time.

$\mathrm{O}$ atleta $\mathrm{A} 2$ também relatou:

Como tinha gente [no jogo] que não veio nos treinos, a gente não conseguia fazer isso [criar espaços] porque às vezes ficavam três jogadores juntos invés de rodar, sair do lugar. Teve um treino que você passou que a gente tinha que passar a bola e sair do lugar que a gente estava, isso ajudou bastante (A 4 concorda com a cabeça), só que como teve gente que não foi no treino, esses passavam a bola e continuava no mesmo lugar.

Ao ser questionado sobre o significado do termo "expressivo", o atleta A3 explicou se referenciar a participação em treinos. $\mathrm{O}$ jogo mencionado pelo atleta $\mathrm{A} 2$ se refere a um amistoso realizado pela equipe no período de realização desta pesquisa, entretanto, este jogo não fez parte deste estudo. Nesse jogo participaram alguns atletas que não compareceram aos treinos de implementação. Ressaltamos que o estudo se passou em um contexto de participação esportiva, onde há menores exigências de presença em treinos e resultado competitivo. Com isso, alguns participantes não participaram de todas as oito sessões, o que se torna um fator limitante da pesquisa. Entretanto, os resultados mostraram que os atletas que participaram com maior frequência dos treinos perceberam diferenças entre aqueles que foram menos presentes. De fato, os participantes mais frequentes tiveram maior contato com os JCA e, consequentemente, maiores oportunidades de vivenciar o ambiente de jogo, o que facilita o desenvolvimento de uma aprendizagem significativa. Segundo Scaglia et al. (2013), a aprendizagem significativa emerge da interação entre o ambiente de aprendizagem (constituído pelos objetivos, conteúdos, procedimentos pedagógicos, entre outros aspectos) e o ambiente de jogo (natureza da atividade, satisfação do jogador e as propriedades reguladoras), exigindo competências essenciais dos jogadores.

\section{Conclusões}

O objetivo deste estudo foi conhecer as opiniões de atletas e treinador sobre o treinamento de iniciação e formação no basquetebol baseado no método do jogo de ataque por conceitos. Os resultados apontaram que os participantes perceberam que o método é motivante e facilita a compreensão da lógica do jogo. Os participantes entenderam que o tempo disponibilizado para o aprendizado dos conceitos apresentados foi curto, o que pode ter sido agravado pelo fato de o conteúdo ser completamente novo para os atletas. Aavaliamos que os JAC podem ser utilizados em programas de ensino do basquetebol no Brasil, com vantagens importantes sobre abordagens tradicionais de ensino-treino.

\section{REFERÊNCIAS}

Araújo, D. y Bourbousson, D. (2016). Theoretical perspectives on interpersonal coordination for team behaviour. In P. Passos, K. Davids, \& J. Y. Chow (Eds.), Interpersonal coordination and performance in social systems (pp. 126139). London: Routledge.

Araújo, D., Davids, K., \& Hristovski, R. (2006). The ecological dynamics of decision making in sport. Psychology of Sport and Exercise, 7, 653-676. doi: https://www.doi.org/10.1016/j.psychsport.2006.07.002 
Araújo, D., Hristovski, R., Seifert, L., Carvalho, J. y Davids, K. (2019). Ecological cognition: expert decision-making behaviour in sport. International Review of Sport and Exercise Psychology, 12(1), 1-25. doi: https://www.doi.o $\mathrm{rg} / 10.1080 / 1750984 X .2017 .1349826$

Bayer, C. (1994). O ensino dos desportos colectivos. Lisboa: Dinalivro.

Berry, J., Abernethy, B. y Côté, J. (2008). The contribution of structured activity and deliberate play to the development of expert perceptual and decision-making skill. Journal of Sport and Exercise Psychology, 30(6), 685-708. doi: h ttps://www.doi.org/10.1123/jsep.30.6.685

Bompa, T. O. (2002). Periodização: teoria e metodologia do treinamento ( $4^{\mathrm{a}}$ ed.). São Paulo: Phorte.

Braun, V.y Clarke, V. (2006). Using thematic analysis in psychology. Qualitative Research in Psychology, 3(2), 77-101. doi: https://www.doi.org/10.1191/1478088706qp063oa

Braun, V., Clarke, V. y Weate, P. (2016). Using thematic analysis in sport and exercise research. In B. Smith y A. C. Sparkes (Eds.), Routledge handbook of qualitative research in sport and exercise (pp. 191-205). London: Routledge. doi: https://www.doi.org/10.4324/9781315762012.ch15

Bunker, D. J. y Thorpe, R. D. (1982). A model for the teaching of games in secondary school. Bulletin of Physical Education, 18(1), 5-8.

Côté, J., Baker, J. y Abernethy, B. (2003). From play to practice: A developmental framework for the acquisition of expertise in team sport. In J. Starkes y K. A. Ericsson (Eds.), Expert performance in sports: Advances in research on sport expertise (pp. 89-113). Champaign,: Human Kinetics.

Côté, J., Baker, J. y Abernethy, B. (2007). Practice and play in the development of sport expertise. In G. Tenenbaum \& R. C. Eklund (Eds.), Handbook of Sport Psychology (pp. 184-202). Hoboken: John Wiley \& Sons.

Creswell, J. W. (2010). Projeto de pesquisa: métodos qualitativo, quantitativo e misto. Porto Alegre: Artmed.

Deci, E. L. y Ryan, R. M. (1985). Intrinsic motivation and self-determination in human behavior. New York: Plenum.

DiFiori, J. P., Brenner, J. S., Comstock, D., Côté, J., Güllich, A., Hainline, B., y Malina, R. (2017). Debunking early single sport specialisation and reshaping the youth sport experience: an NBA perspective. British Journal of Sports Medicine, 51(3), 142-143. doi: https://www.doi.org/10.1136/bjsports-2016-097170

DiFiori, J. P., Güllich, A., Brenner, J. S., Côté, J., Hainline, B., Ryan, E. y Malina, R. M. (2018). The NBA and youth basketball: recommendations for promoting a healthy and positive experience. Sports Medicine, 48(9), 20532065. doi: https://www.doi.org/10.1007/s40279-018-0950-0

Ferreira, H. B., Galatti, L. R. y Paes, R. R. (2005). Pedagogia do Esporte: considerações pedagógicas e metodológicas no processo de ensino e aprendizagem do basquetebol. In R. R. Paes \& H. F. Balbino (Eds.), Pedagogia do Esporte: contextos e perspectivas (pp. 126-136). Rio de Janeiro: Guanabara Koogan.

Ford, P. R., Yates, I., y Williams, A. M. (2010). An analysis of practice activities and instructional behaviours used by youth soccer coaches during practice: Exploring the link between science and application. Journal of Sports Sciences, 28(5), 483-495. doi: https://www.doi.org/10.1080/02640410903582750

Fraser-thomas, J., Côté, J, y Deakin, J. (2005). Youth sport programs: an avenue to foster positive youth development. Physical Education and Sport Pedagogy, 10(1), 19-40. doi: https://www.doi.org/10.1080/1740898042000334 890

Galatti, L. R., Bettega, O. B., Paes, R. R., Reverdito, R. S., Seoane, A. M. y Scaglia, A. J. (2017). O ensino dos jogos esportivos coletivos: avanços metodológicos dos aspectos estratégico-tático-técnicos. Pensar a Prática, 20(3), 639-654. doi: https://www.doi.org/10.5216/rpp.v20i3.39593

Galatti, L. R., Côté, J., Reverdito, R. S., Allan, V., Seoane, A. M. y Paes, R. R. (2016). Fostering elite athlete development and recreational sport participation: A successful club environment. Motricidade, 2(3), 20-31. doi: https://w ww.doi.org/10.6063/motricidade.6099

Galatti, L. R., Ferreira, H. B., Silva, Y. P. G. y Paes, R. R. (2008). Pedagogia do esporte: procedimentos pedagógicos aplicados aos jogos esportivos coletivos. Conexöes, 6(especial), 397-408. doi: https://www.doi.org/10.20396/c onex.v6i0.8637843 
Galatti, L. R., Paes, R. R. y Seoane, A. M. (2009). Pedagogía del deporte y juegos deportivos colectivos: el juego como elemento en los programas a largo plazo de escuelas deportivas. Revista de Educación Física: Renovar La Teoría y Practica., 11(3), 13-18.

Galatti, L. R., Reverdito, R. S., Scaglia, A. J., Paes, R. R. y Seoane, A. M. (2014). Pedagogia do Esporte: Tensão na ciência e o ensino dos jogos esportivos coletivos. Revista Da Educacao Fisica, 25(1), 153-162. doi: https://ww w.doi.org/10.4025/reveducfis.v25i1.21088

Galatti, L. R., Seoane, A. M. y Paes, R. R. (2012). Pedagogia do esporte e basquetebol: aspectos metodológicos para o desenvolvimento motor e técnico do atleta em formação. Arquivos Em Movimento, 8(2), 79-93.

Gray, S. y Sproule, J. (2011). Developing pupils' performance in team invasion games. Physical Education and Sport Pedagogy, 16(1), 15-32. doi: https://www.doi.org/10.1080/17408980903535792

Greco, P. J. (1998). Iniciação Esportiva Universal: metodologia da iniciação esportiva na escola e no clube (2ª ed.). Belo Horizonte: UFMG.

Greco, P.J. (2006). Conhecimento técnico-tático: o modelo pendular do comportamento e da ação tática nos esportes coletivos. Revista Brasileira de Psicologia Do Esporte e Do Exercício, 0, 107-129.

Hennink, M. M. (2014). Focus group discussions: understanding qualitative research. New York: Oxford University Press.

Huizinga, J. (1999). Homo ludens: o jogo como elemento da cultura. São Paulo: Perspectiva.

Ibañez, S. J., Garcia-Rubio, J., Gómez, M.-Á. y Gonzalez-Espinosa, S. (2018). The Impact of Rule Modifications on Elite Basketball Teams' Performance. Journal of Human Kinetics, 64(1), 181-193. doi: https://www.doi.org/1 0.1515/hukin-2017-0193

Kirk, D. y MacPhail, A. (2002). Teaching Games for Understanding and Situated Learning: Rethinking the BunkerThorpe Model. Journal of Teaching in Physical Education, 21(2), 177-192. doi: https://www.doi.org/10.1123 /jtpe.21.2.177

Leonardo, L., Scaglia, A. J. y Reverdito, R. (2009). O ensino dos esportes coletivos: metodologia pautada na família dos jogos. Motriz, 15(2), 236-246.

Light, R., \& Tan, S. (2006). Culture, embodied experience and teachers' development of TGfU in Australia and Singapore. European Physical Education Review, 12(1), 99-117. doi: https://www.doi.org/10.1177/1356336X 06060659

Machado, G. V., Galatti, L. R. y Paes, R. R. (2012). Seleção de conteúdos e procedimentos pedagógicos para o ensino do esporte em projetos sociais: reflexões a partir dos jogos esportivos coletivos. Motrivivência, 24(39), 164-176. doi: https://www.doi.org/10.5007/2175-8042.2012v24n39p164

Maricone, L. M., Santos, Y. Y. S., Pérez, B. L. y Galatti, L. R. (2016). Pedagogia do Esporte: uma proposta de iniciação em basquetebol a partir de conceitos do jogo pautados no método da Federação Espanhola. Corpoconsciência, 20(3), 57-67.

Menezes, R. P., Marques, R. F. R. y Nunomura, M. (2014). Especialização esportiva precoce e o ensino dos jogos coletivos de invasão. Movimento, 20(1), 351-373. doi: https://www.doi.org/10.22456/1982-8918.40200

Morales, J. C. P. y Greco, P. J. (2007). A influência de diferentes metodologias de ensino-aprendizagem-treinamento no basquetebol sobre o nível de conhecimento tático processual. Revista Brasileira de Educação Física e Esporte, 21(4), 291-299. doi: https://www.doi.org/10.1590/S1807-55092007000400004

Moreira, A. y Paes, F. O. (2011). Basquetebol. In M. T. S. BÖHME (Ed.), Esporte infantojuvenil: treinamento a longo prazo e talento esportivo (pp. 367-390). São Paulo: Phorte.

Paes, R. R. (2001). Educação física escolar: o esporte como conteúdo pedagógico no ensino fundamental. Canoas: Ulbra.

Patton, M. Q. (2002). Two Decades of Developments in Qualitative Inquiry. Qualitative Social Work: Research and Practice, 1(3), 261-283. doi: https://www.doi.org/10.1177/1473325002001003636

Pelletier, L. G., Tuson, K. M., Fortier, M. S., Vallerand, R. J., Briére, N. M. y Blais, M. R. (1995). Toward a New Measure of Intrinsic Motivation, Extrinsic Motivation, and Amotivation in Sports: The Sport Motivation Scale (SMS). Journal of Sport and Exercise Psychology, 17(1), 35-53. doi: https://www.doi.org/10.1123/jsep.17.1.35 
Reverdito, R. S., Galatti, L. R., Scaglia, A. J. y Paes, R. R. (2020). Youth participation in sport in a context of vulnerability: perception of positive and negative experiences. E-Balonmano.Com: Journal of Sports Science / Revista de Ciencias Del Deporte, 16(1), 55-66. Recuperado de: http://proxy.uqtr.ca/login.cgi?action=login\&u=uqtr\&db=ebsco\&ezurl=http://search.ebscohost.com/log in.aspx?direct $=$ true\& $\mathrm{db}=$ s3h\&AN $=142076760 \&$ site $=$ ehost-live

Rigon, T. A., Novaes, R. B. y Tsukamoto, M. H. C. (2020). A elaboração de uma matriz de referência para o ensino dos jogos esportivos coletivos. Corpoconsciência, 24(2), 172-186. Recuperado de: https://periodicoscientificos. ufmt.br/ojs/index.php/corpoconsciencia/article/view/10713

Rivero, J. C. (s.d.). Aspectos generales del juego sin balón. Espanha: Club del entrenador/Federación Española de Baloncesto. Recuperado de http://clubdelentrenador.feb.es/articulos/242.pdf

Robles, M. T. A., Benito, P. J., Fuentes-Guerra, F. J. G. y Rodríguez, J. R. (2013). Fundamentos pedagógicos de la enseñanza comprensiva del deporte: Una revisión de la literatura. Cultura, Ciencia y Deporte, 8(23), 137-146. doi: https://www.doi.org/10.12800/ccd.v8i23.300

Ryan, R. M. y Deci, E. L. (2000). Self-determination theory and the facilitation of intrinsic motivation, social development, and well-being. American Psychologist, 55(1), 68-78. doi: https://www.doi.org/10.1037110003 -066 X.55.1.68

Scaglia, A. J., Reverdito, R. y Galatti, L. (2013). Ambiente de jogo e ambiente de aprendizagem no processo de ensino dos jogos esportivos coletivos: desafios no ensino e na aprendizagem dos jogos esportivos coletivos. In J. Nascimento, V. Ramos y F. Tavares (Eds.), Jogos Desportivos: formação e investigação (p. 512). Florianópolis: UDESC.

Scaglia, A. J., Reverdito, R., Leonardo, L. y Lizana, C. J. R. (2013). O ensino dos jogos esportivos coletivos: as competências essenciais e a lógica do jogo em meio ao processo organizacional sistêmico. Movimento, 19(4), 227-249. doi: https://www.doi.org/10.22456/1982-8918.37893

Silva, J. M. G. (2007). O ensino dos jogos desportivos colectivos. Perspectivas e tendências. Movimento, 4(8), 19-27. doi: https://www.doi.org/10.22456/1982-8918.2373

Silva, P., Esteves, P. T., Correia, V., Davids, K., Araújo, D. y Garganta, J. (2015). Effects of manipulations of player numbers vs. field dimensions on inter-individual coordination during small-sided games in youth football. International Journal of Performance Analysis in Sport, 15(2), 641-659. doi: https://www.doi.org/10.1080/24 748668.2015.11868821

Silva, P., Garganta, J., Araújo, D., Davids, K. y Aguiar, P. (2013). Shared Knowledge or Shared Affordances? Insights from an Ecological Dynamics Approach to Team Coordination in Sports. Sports Medicine, 43(9), 765-772. doi: https://www.doi.org/10.1007/s40279-013-0070-9

Silva, R. M. P., Galatti, L. R. y Paes, R. R. (2010). Pedagogia do esporte e iniciação esportiva tardia: perspectivas a partir da modalidade basquetebol. Pensar a Prática, 13(1), 1-15. doi: https://www.doi.org/10.5216/rpp.v13i1.7629

Smith, B. y Sparkes, A. (2016). Interviews: Qualitative Interviewing in the Sport and Exercise Sciences. In B. Smith \& A. Sparkes (Eds.), Routledge Handbook of Qualitative Research in Sport and Exercise (pp. 103-123). Londres: Routledge.

Travassos, B., Araújo, D., Davids, K., Vilar, L., Esteves, P. y Vanda, C. (2012). Informational constraints shape emergent functional behaviours during performance of interceptive actions in team sports. Psychology of Sport and Exercise, 13(2), 216-223. doi: https://www.doi.org/10.1016/j.psychsport.2011.11.009

Tripp, D. (2005). Pesquisa-ação: uma introdução metodológica. Educação e Pesquisa, 31(3), 443-466. doi: https://w ww.doi.org/10.1590/S1517-97022005000300009

Turner, A. y Martinek, T.J. (1995). Teaching for understanding: A model for improving decision making during game play. Quest, 47(1), 44-63. doi: https://www.doi.org/10.1080/00336297.1995.10484144

Vaughan, J., Mallett, C. J., Davids, K., Potrac, P. y López-Felip, M. A. (2019). Developing creativity to enhance human potential in sport: a wicked transdisciplinary challenge. Frontiers in Psychology, 10 (September), 1-16. doi: http s://www.doi.org/10.3389/fpsyg.2019.02090 
Werner, P. y Almond, L. (1990). Models of Games Education. Journal of Physical Education, Recreation \& Dance, 61(4), 23-30. doi: https://www.doi.org/10.1080/07303084.1990.10606501

Zoilo, Á. G. (2008). El juego de ataquepor conceptos. Espanha: Club del entrenador/Federación Española de Baloncesto. Recuperado de https://www.clubdelarbitro.com/articulos/344893.pdf 